\title{
Harnessing the heterogeneity of aging
}

\author{
Warren Ladiges $^{\mathrm{a},{ }^{,}}$ \\ ${ }^{a}$ Department of Comparative Medicine, School of Medicine, University of Washington, Seattle, WA 98195, USA.
}

\begin{abstract}
Genetic variation does not entirely address the consistent divergence of healthy and unhealthy aging in heterogeneous and homogeneous mammalian populations. The alteration of gene function through modification of histone DNA infrastructure is a logical extending explanation for this divergence. Since epigenetic alterations are reversible, therapeutic interventions that target the right gene or gene products could reverse aging, at the very least in the population of older people with poor health.
\end{abstract}

Keywords: Heterogeneity of aging, epigenetic alterations, reversing aging

Immortality is not a flesh and blood reality, since death is inevitable. This is an issue that should not require much scientific preponderance. However, it makes sense to focus on staying as healthy as possible with increasing age. Healthy aging is a term used frequently to distinguish aging with relatively few disease complications, in contrast to aging per se, which generally means unhealthy aging and extensive disease complications. So why do some people live longer than others, and some experience good health in their later years compared to others that are plagued with comorbidities?

In order to address this question, several factors need to be considered. Genetic factors are at the forefront of biogerontology research, and rightfully so, as genes code for proteins that respond to stress in specific ways. Variations in genetic sequence can result in different ways of responding to that stress, which may increase or decrease aspects of healthy aging. Rare variants have been identified in centenarians that would appear to be natural therapeutic targets for enhancing healthy aging. However, it seems precarious that aging in humans, a heterogeneous population, is similar to aging in more homogeneous populations. For example, certain inbred strains of laboratory mice have been shown to outlive other strains, yet within each strain, there is the same biological divergence of healthy and unhealthy aging groups with increasing chronological age as seen in humans. This would suggest there are additional factors to explain why some people

\footnotetext{
* Corresponding author: Warren Ladiges

Mailing address: Department of Comparative Medicine, School of Medicine, University of Washington, Seattle, WA 98195, USA. E-mail: wladiges@uw.edu

Received: 25 February 2021 / Accepted: 11 March 2021
}

experience healthy aging and others age poorly.

One possible explanation for this observation is epigenetic influence. Epigenesis is the term used to describe the manner in which endogenous or exogenous factors can alter gene function without direct changes in DNA structure. Therefore, epigenesis is a constantly fluid process that controls whether a gene or set of genes, under-performs or over-performs. For example, histone deacetylase (HDAC) is an epigenetic protein that shrinks the histone backbone that supports the DNA helix, thus repressing gene transcription and coding of critical proteins involved in healthy aging. Inhibition of HDAC would thus seem to be a potential therapeutic target to enhance healthy aging. There are likely a multitude of proteins that attack the histone infrastructure that are not yet identified but have a significant influence on gene function at critical times over a lifespan.

The argument that epigenesis can alter gene function to enhance healthy aging or accelerate unhealthy aging is a logical argument but not yet scientifically proven. Extensive research in this area will be needed to determine how the heterogeneity of aging can be harnessed to benefit the health of older people. One very strong rationale for moving in this direction is that epigenetic alterations are reversible up to a point, meaning that therapeutic interventions that target the right gene or gene products could reverse aging. Could this be a path toward longer life in addition to healthier aging? Maybe, but at the very least, it could be the promise of enhancing the lives of those older people experiencing unhealthy aging.

\section{Declaration}

Acknowledgments: Supported by NIH grants R01 AG057381 and R01 AG067193 (PI, Ladiges). 